\title{
Improved Genetic-ant Algorithm for the Optimization of Rapid Prototyping Contour Path
}

\author{
Baoye Liu $^{\mathrm{a}}$, Jianjun Yang ${ }^{\mathrm{b}}$, Shaoshuai Guo ${ }^{\mathrm{c}}$ \\ School of Mechanical Engineering, Qingdao Technological University, Qingdao 266033, China \\ albysd@126.com, ${ }^{\mathrm{b}}$ Corresponding author: yjjdem@163.com, ${ }^{c}$ icomeicontrol@hotmail.com
}

Keywords: Rapid prototyping, Contour path optimization, Improved genetic-ant algorithm, Time distance, Dual integer coding

\begin{abstract}
In rapid prototyping contour scanning process, to reduce the spare travel time, a mathematical model which has considered the minimal time distance is established. The situation that the starting point is different from the end point is analyzed. In the improved genetic-ant algorithm, the dual integer coding is adopted to optimize the contour scanning order and starting point of contour simultaneously, we adopt genetic algorithm to produce initialization pheromone, and realize the fusion of ant colony algorithm and genetic algorithm. In the hybrid algorithm, the new state transition rule is adopted, dynamic volatile coefficient is adopted for pheromone updating, the pheromone and elicitation information are used to choose crossover and mutation positions. The computation result shows that the algorithm can optimize the rapid prototyping contour path effectively.
\end{abstract}

\section{Introduction}

In rapid prototyping, the scanning path contains two categories, filling path and contour path [1]. Information of each layer is got through layers part, and then each contour on the layer is filled out by offset or parallel scanning, a path is formed by joining up all contours in a certain order, which is contour path. Because of the different ways of forming parts, contour path planning has two cases: the starting point and the end point overlap or not.

For the situation that the starting point and the end point overlap, reference [2] regarded this question as generalized traveling salesman problem (GTSP), two level coding is constructed both path and the starting point, and genetic algorithm is used. Reference [3] puts forward the two step optimization algorithm, first according to the contour original starting point to determine the contour processing sequence, and then assign the contour starting point.

In this paper, combining with the laser scanning XY axis operation characteristics, taking time distance as the optimization goal, aiming at the situation that the starting point is different from the end point, we designed the improved genetic-ant algorithm to optimize the contour path.

\section{The Optimal Mathematical Model}

Optimization objective is to reduce the spare travel time in rapid prototyping contour scanning process. Time is not proportional to distance when the speed changes. The concept of time distance is used which means the distance of the shortest time. In spare travel, XY-axis scanning mechanism can run at maximal speed, and the starting point is different from the end point, so the spare travel time that starting from the end point $P_{i}\left(x_{i}^{e}, y_{i}^{e}\right)$ of the contour $i$ to the starting point $P_{j}\left(x_{j}^{s}, y_{j}^{s}\right)$ of the contour $j$, depends on the value of $\max \left\{\operatorname{fabs}\left(x_{j}^{s}-x_{i}^{e}\right), \operatorname{fabs}\left(y_{j}^{s}-y_{i}^{e}\right)\right\}$, that is the time distance [2].

Assume the origin of laser scanning $P_{0}=\left(x_{0}, y_{0}\right)$, the objective function as follows.

$$
\begin{aligned}
\min f= & \max \left\{\operatorname{fabs}\left(x_{1}^{s}-x_{0}\right), \text { fabs }\left(y_{1}^{s}-y_{0}\right)\right\}+\sum_{i=1}^{n-1} \max \left\{\operatorname{fabs}\left(x_{i+1}^{s}-x_{i}^{e}\right), \text { fabs }\left(y_{i+1}^{s}-y_{i}^{e}\right)\right\}, \\
& +\max \left\{\operatorname{fabs}\left(x_{0}-x_{n}^{e}\right), \text { fabs }\left(y_{0}-y_{n}^{e}\right)\right\}
\end{aligned}
$$


where, $n$ is the amounts of contour, $x_{i}^{s}, y_{i}^{s}$ is the starting point coordinate of contour $i, x_{i}^{e}, y_{i}^{e}$ is the end point coordinate of contour $i$.

In the process of improved genetic-ant algorithm, the optimization objective direction of the problem should be in accordance with the direction that the value of fitness function becomes larger. This paper uses the following fitness function $F$.

$$
F=1 / f \text {. }
$$

\section{Algorithm Design}

Genetic algorithm has strong global searching capability, but the feedback information is not well utilized, leading to low solution efficiency. Ant colony algorithm converges in the optimal path by the pheromone accumulation and updating, but lacks of initial pheromone, leading to slowly convergence and easily falling into the local optimal solution [4]. Therefore, we adopt improved algorithm that first uses genetic algorithm to generate several optimal solutions and generate the initial pheromone according to the way of reference [5], and then fuses the ant colony algorithm with genetic algorithm.

Coding Design. According to the characteristics of optimization problem, this paper adopts dual integer coding. The first line indicates the order of scanning contour in the path. The second line indicates the position of each contour starting point. The end point is associated with the starting point, the contour filling way, the characteristics of contour and the laser pulse width. There are many filling ways in the contour internal scanning. This paper adopts the most basic scanning way that is the $\mathrm{X}$ axis parallel scanning way, the starting point corresponds to the $\mathrm{Y}$ axis maximal or minimal value, the end point is decided by contour height and the laser pulse width, which is the $\mathrm{Y}$ axis minimal or maximal value. In Fig. 1 , the starting point of contour 2 can take any one in the point set $\{1$, $2,3,4\}$, if the starting point is 2 , the end point can be only one in the point set $\{1,4\}$ (depending on the contour height and the laser pulse width), the starting point of contour 3 can take any one in the point set $\{1,2\}$, the end point is another, the point (3) and (4) can't be the starting point or the end point.

An example of processing path as shown in Fig.1, and the chromosome $\mathrm{C}_{1}$ is corresponding to it.

$$
C_{1}=\left[\begin{array}{lllll}
2 & 3 & 4 & 1 & 5 \\
2 & 1 & 1 & 1 & 2
\end{array}\right] \text {. }
$$

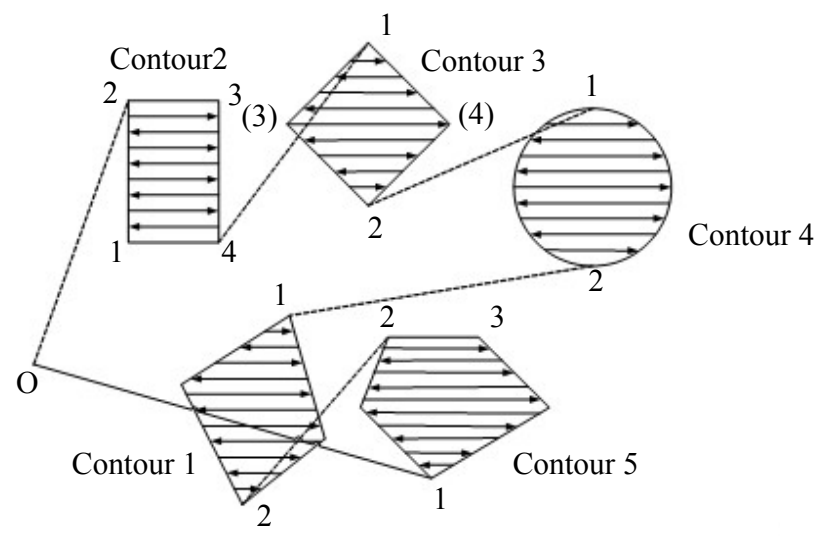

Fig.1 Example of processing path

Ant State Transition Rule. The initial solution selection often guides the ant colony convergence in local optimal solution. In order to reduce the decisive role of initial solution, in the process of ant choosing nodes, we should reasonably use historical information, and then increase the search space as much as possible.

In view of the above analysis, we construct the following searching mechanism, depending on which ant $k$ chooses the node $j$. 


$$
j= \begin{cases}J_{1}=\arg \max _{j \in \text { allowed }_{k}}\left(\tau_{i j}^{\alpha} \cdot \eta_{i j}^{\beta}\right) & 0 \leq q<q_{1}, \\ J_{2}\left(p_{i j}^{k}\right): p_{i j}^{k}=\frac{\tau_{i j}^{\alpha} \cdot \eta_{i j}^{\beta}}{\sum_{l \in \text { allowed }_{k}} \tau_{i j}^{\alpha} \cdot \eta_{i l}^{\beta}} & q_{1} \leq q \leq q_{2}, \\ J_{3}: \text { Random selection } & q_{2}<q \leq 1\end{cases}
$$

where, allowed al $_{k}=\{0,1, \cdots, n-1\}$ is the node set that are allowed to select by ant $k$ in the next step, $q$ is the random decimal number between 0 and $1, q_{1}, q_{2}$ are adjustment parameters, $0<q_{1}<q_{2}<1, \tau_{i j}$ is the pheromone content on the edge $(i, j), \eta_{i j}$ is the visibility of edge $(i, j)$, reflects the heuristic information from the node $i$ transferred to the node $j, P_{i j}^{k}$ is the ant $k$ transferring probability from the node $i$ to choice the node $j, \alpha, \beta$ are two parameters, which respectively reflect the relative importance of the pheromone and the heuristic information in the process of ant choosing node.

Pheromone Updating Mechanism. Based on the max-min ant system, we update the pheromone and dynamically adjust volatile coefficient $\rho$, as follows.

$$
\begin{aligned}
& \tau_{i j}=(1-\rho) \cdot \tau_{i j}+\Delta \tau, \\
& \rho=0.05+N_{c} /\left(20 \times N_{c \text { max }}\right),
\end{aligned}
$$

where, $\Delta \tau$ is the incremental pheromone, $\rho$ is the volatile coefficient, for combinatorial optimization problems, in the initial stage needs to be slightly larger for enhancing the exploration capability of algorithm, while in the late needs to be smaller to increase the algorithm focused search capability, in this paper, the values range are interval $(0.05,0.15), N_{c}$ is the current iteration number, $N_{c \max }$ is the maximal iteration number.

Choose Operation. In this paper, the choose operation is identified by the fitness function value and the pheromone content, the choose probability $P_{i}$ of individual $i$ is as follows.

$$
P_{i}=\tau_{i}^{\kappa} F_{i}^{\lambda} / \sum_{i=1}^{n_{p}}\left(\tau_{i}^{\kappa} F_{i}^{\lambda}\right)
$$

where, $n_{\mathrm{p}}$ is the amounts of paths, $\kappa$ is the important factor of pheromone, $\lambda$ is the important factor of fitness, $\tau_{i}$ is the pheromone content on the $i$ th path.

Selection of Crossover and Mutation Position. In crossover and mutation operation of genetic algorithm, crossover and mutation position is randomly selected, which improves the global searching capability, but increases the number of invalid operation. Therefore, adopting the pheromone content between node $i$ and node $j$ and the length of edge $s$ which is formed by node $i$ and node $j$, we establish the following select mechanism.

$$
s=\left\{\begin{array}{cc}
\mathrm{S}_{1}=\operatorname{argmax}\left(d_{\mathrm{s}}^{\gamma} / \tau_{\mathrm{s}}^{\delta}\right) & 0 \leq q \leq q_{3} \\
\mathrm{~S}_{2}: \text { Random selection } & q_{3}<q \leq 1
\end{array},\right.
$$

where, $q_{3}$ is adjustment parameters, $0<q_{3}<1, d_{s}$ is the length of edge $s, \gamma$ and $\delta$ are two parameters, which respectively reflect the relative importance of the pheromone and length on the edge $s$ in the selection of crossover and mutation position. This operation increases the probability that the long edge or the edge of less pheromone is the crossover and mutation position.

Crossover Operation. To increase the diversity of population, we design a variety of crossover methods. This paper randomly selects one of the following two crossover methods: 1) the contour order cross each other, the corresponding starting point is unchanged. 2) The starting point cross each other, the corresponding contour order is unchanged. The contour order crossover adopts partially mapped crossover. The starting point adopts uniform crossover during the corresponding contour starting point optional range.

Mutation Operation. To prevent the genetic-ant algorithm from sinking into local optimal, we design a variety of mutation methods. This paper randomly selects one of the following two mutation methods: 1) the contour order mutates, the corresponding starting point is unchanged. 2) The starting 
point mutates, the corresponding contour order is unchanged. The contour order mutation adopts reverse mutation. The starting point mutation adopts non-uniform mutation during the corresponding contour starting point optional range.

\section{Optimization Example}

Based on this algorithm, we use Visual $\mathrm{C}++$ to make up optimization program, and optimize one contour path in SLA, there are 30 contours in one layer. With genetic algorithm, ant colony algorithm and improved genetic-ant algorithm optimizing this question, the results are showed in Table 1 . When using the improved genetic-ant algorithm, the time distance decreases $37.9 \%$ and $31.2 \%$, compared with genetic algorithm and ant colony algorithm, respectively.

Table 1 Comparison between optimization results

\begin{tabular}{|c|c|l|}
\hline $\begin{array}{c}\text { Optimal } \\
\text { method }\end{array}$ & $\begin{array}{c}\text { Time distance } \\
(\mathrm{mm})\end{array}$ & \multicolumn{1}{|c|}{ Contour order (Starting point) } \\
\hline $\begin{array}{c}\text { Genetic } \\
\text { algorithm }\end{array}$ & 763 & $\begin{array}{l}14(1), 13(1), 12(1), 27(2), 26(1), 21(1), 20(2), 18(2), 19(3), 17(1), 11(1), 10(2), \\
9(2), 8(3), 7(2), 5(2), 6(3), 4(2), 16(2), 15(1), 28(2), 3(3), 2(3), 1(2), 30(1), 29(3), \\
25(1), 24(1), 23(4), 22(2)\end{array}$ \\
\hline $\begin{array}{c}\text { Ant colony } \\
\text { algorithm }\end{array}$ & 689 & $\begin{array}{l}30(1), 29(2), 25(1), 24(1), 21(1), 20(1), 18(2), 19(4), 17(1), 11(2), 10(2), 9(2), \\
4(1), 16(2), 15(2), 8(2), 7(2), 5(2), 6(2), 14(1), 13(2), 12(1), 27(4), 26(2), 28(2), \\
3(3), 2(3), 1(3), 23(3), 22(2)\end{array}$ \\
\hline $\begin{array}{c}\text { Improved } \\
\text { genetic-ant } \\
\text { algorithm }\end{array}$ & 474 & $\begin{array}{l}21(1), 20(2), 18(1), 19(3), 17(2), 11(2), 10(1), 9(3), 8(1), 7(2), 5(2), 6(4), 4(2), \\
16(1), 15(1), 14(3), 13(3), 12(3), 27(1), 26(2), 28(1), 3(3), 2(3), 1(3), 30(1), \\
29(1), 25(2), 24(2), 23(3), 22(2)\end{array}$ \\
\hline
\end{tabular}

\section{Conclusions}

In this paper, we take the time distance as the optimization objective, and analysis the coding style when the starting point doesn't coincide with the end point. Using improved genetic-ant algorithm to solve problem. According to the actual need of problem, we adopt dual integer coding. In order to improve the algorithm optimal performance, we use genetic algorithm to generate initial solution firstly, and then fuses the ant colony algorithm with genetic algorithm, adopt fitness and pheromone to jointly control the selecting operation, select crossover and mutation position by pheromone and heuristic information, dynamically adjust pheromone update mechanism. Comparing with other algorithms indicates that this algorithm is feasible.

\section{References}

[1] J. P. Kruth, M. C. Leu and T. Nakagawa: Annals of CIRP Vol.47 (1998), p.525

[2] W. D. Yang, R. H. Tan and Y. N. Yan: Journal of Computer-aided Design \& Computer Graphics Vol.17 (2005), p.2179 (in Chinese)

[3] G. X. Yu, Y. C. Li and D.C. Ding: Journal of Xi' an Jiao Tong University Vol.38 (2004), p.39 (in Chinese)

[4] J. R. Xu, Y. Li and H. T. Liu: Computer Applications Vol.28 (2008), p.2084 (in Chinese)

[5] C. X. Wang and X. N. Guo: Machine Tool \& Hydraulics Vol.39 (2011), p.43 (in Chinese) 